\title{
SHIFT OF THE COLLECTIVE MODE OF POLARIZED GRAPHENE ON A SUBSTRATE USING SPIN-SENSITIVE RESPONSE THEORY
}

\author{
D. Kreil, M. Haslhofer, and H.M. Böhm \\ Institute for Theoretical Physics, Johannes Kepler University, Altenbergerstraße 69, 4040 Linz, Austria \\ Email: dominik.kreil@jku.at
}

Received 7 June 2018; revised 6 July 2018; accepted 15 October 2018

\begin{abstract}
The growing precision of optical and scattering experiments necessitates a better understanding of the influence of damping onto the collective mode of sheet electrons. As spin-polarized systems are of particular interest for spintronic applications, we here report spin-sensitive linear response functions of graphene, which give access to chargeand spin-density related excitations. We further calculate the reflectivity of graphene on an $\mathrm{SiO}_{2}$ surface, a setup used in s-wave scanning near-field microscopy. Increasing the partial spin-polarization of the graphene charge carriers leads to a significant broadening and shift of the plasmon mode, due to single-particle interband transitions of the minority spin carriers. We also predict an antiresonance in the longitudinal magnetic response function, similar to that of semiconductor heterostructures.
\end{abstract}

Keywords: graphene, s-SNOM, collective modes, plasmom, reflectivity

PACS: 68.65.Pq, 05.30.Fk, 71.45.Gm, 71.45.-d, 71.10.-w, 71.10.Ca

\section{Introduction}

Although the existence of purely two-dimensional (2D) materials is prohibited by long-range thermal fluctuations [1], in 2004 Geim and Novoselov [2] produced monoatomic thin graphite layers, now famous as 'graphene'. (Anharmonically coupled streching/bending modes [3-5] prevent the instability; the resulting rippling is avoided by placing the sheet on a flat support material.) Graphene's honeycomb structure implies many captivating properties, e.g. despite its thinness, a mechanical strength 200 times that of steel. The large carrier mobility results in excellent thermal and electric conductivity, dynamically tunable by chemical doping or an applied gate voltage [6]. Doubtless, graphene is a promising candidate for high-speed and optoelectronic devices [8, 8].
In addition, graphene holds the most intriguing potential for spintronic applications: the spin-orbit coupling (SOC) allows the development of appliances where, due to their interplay, spin and charge currents can be manipulated simultaneously, offering the perspective of novel logic and memory devices. Clearly, this requires a thorough theoretical understanding of graphene's spin-resolved properties. Of particular interest is the collective behaviour of the charge carriers: effective, spin-dependent interactions and correlations between the charge carriers (electrons or holes) have manifest fingerprints in the excitation spectrum, accessible experimentally. Specifically, light scattering from surfaces using scanning near-field optical microscopy (s-SNOM) has provided accurate data on graphene, pioneered by Fei et al. [9] in the mid-infrared, and later extended to the teraherz range [10, 11]. 
Graphene's valence- and conduction-band energies touch at the 6 corner points of the Brillouin zone (BZ). Half of them are equivalent (as two atoms are in the unit cell), and referred to as $K$ and $K^{\prime}$ points. In their vicinity electrons and holes behave as massless Dirac fermions [12] with a linear energy dispersion, contrary to the quadratic one of conventional 2D electron liquids (2DELs) in semiconductor layers. The single-particle excitations then form continua ('particle-hole bands', PHBs) with linear boundaries; they offer prominent decay channels ('Landau damping' [13]) for the plasmon. The vanishing gap brings about another crucial difference to the standard interface 2DELs: interband transitions lead to damping at much shorter wavelengths than the intraband PHB. Consequently, graphene's plasmon is much stronger influenced by a spin polarization of the system, because the interband $\mathrm{PHB}$ edge is drastically decreased with increasing spin imbalance.

Experimentally, 2DELs with a different amount of $\uparrow$ and $\downarrow$ spins have been realized (various methods being reviewed in [14]). For such systems a long-lived 'spin-plasmon' (the longitudinal magnon) was predicted [15]. Placing such a spinimbalanced 2DEL between coupled (spin-torque) nanomagnets would enable tuning of their coupling via controlling the spin populations. With the prominent electron layers realized in GaAsGaAlAs heterostructures this intriguing idea does not work, as correlations lower the spin-plasmon peak [16].

In graphene, where the touching Dirac cones imply a richer excitation spectrum even in the simple linear-dispersion model, they prevent a straightforward generalization of these predictions. Therefore, it is highly interesting to study the effect from scratch. To the best of our knowledge, the dielectric response of partially spin-polarized graphene has not yet been investigated. In this work, we derive the partial Lindhard functions from spin-sensitive linear response theory. For ease of reading, the spin density ( $\propto$ the magnetization's $z$ component) in this context is simply referred to as 'spin', i.e. 'spin-spin response' stands for 'spin-density - spin-density response'. We performed the first Random Phase Approximation (RPA) calculations for single-layer graphene with a spin imbalance, and present results for the density-density (or charge-charge) response, the spin-spin response, as well as for the densityspin response. The latter describes magnetic excitations caused by electric perturbations and vice versa, resulting not from the SOC but the Coulomb interaction and Pauli exclusion.

In addition, we apply our dielectric function to determine the reflectivity of graphene on a $\mathrm{SiO}_{2}$ substrate as studied in s-SNOM experiments [9]. When, at a sufficiently high doping, the plasmon energy reaches that of optical phonons in the substrate, the coupling between the modes causes the dispersions to 'repel' each other. We investigate how this is affected by a spin imbalance.

This work is organized as follows. We first address in Section 2 the fundamental ambiguity of defining a collective mode's precise location if damping is significant. In Section 3, after briefly reviewing the energy bands and presenting the spin-dependent formalism (3.1.), we derive the partial Lindhard functions for spin $\sigma$ fermions (3.2.) and the resulting RPA response functions (3.3.). The reflectivity of spin-imbalanced graphene on $\mathrm{SiO}_{2}$ is studied in Section 4, followed by a critical discussion of our results in Section 5. All calculations are done for zero temperature $T=0$, the majority of spins are denoted without loss of generality as $\uparrow$.

\section{Collective modes}

Collective excitations of many particles are characterized by their in-phase movement. Charge carriers in solids have additional degrees of freedom, e.g. a spin, and possibly a 'pseudospin' due to different valleys (band structure minima with the same energy at different points of the $\mathrm{BZ}$ as in graphene's $K, K^{\prime}$ points). In such multicomponent systems, in addition to the overall collective mode of the density, various subspecies may oscillate with opposite phases. The full density mode is the plasmon (with an in-phase oscillation of all spins). The longitudinal mode where $\uparrow$-spins collectively move against $\downarrow$-spins is referred to as the 'spin-plasmon' [15].

Long-lived collective excitations are mathematically found from poles in response functions, equivalent to peaks in the scattering cross section. However, if damping and drag forces are present, their determination becomes ambiguous [17]. We 
exemplify this for the Drude model for classical charge carriers [18]: $\epsilon_{\mathrm{D}}(\omega)=1-\omega_{\mathrm{pl}}^{2} / \omega(\omega+\mathrm{i} \bar{\eta})$, with the classical plasmon frequency $\omega_{\mathrm{pl}}$ and damping parameter $\bar{\eta} \equiv \eta \omega_{\mathrm{pl}}$. Measurements [19] of the graphene optical transmission and reflection coefficient are well described by this model with $\eta=0.007$. From a spectroscopic perspective, the plasmon is best defined [20] as the complex zero of the complex dielectric function $\epsilon\left(\omega_{1}+i \omega_{2}\right)$. In the Drude model damping shifts the observed mode towards lower energies $\omega_{1} / \omega_{\mathrm{pl}}=\left(1-\eta^{2} / 4\right)^{1 / 2}$, with $\omega_{2}=\eta / 2$.

By contrast, scattering experiments (e.g. electron energy loss spectroscopy (EELS) [21, 22]) probe the loss function $\operatorname{Im} \epsilon^{-1}(\omega)$, proportional to the scattering cross-section. Correspondingly, the plasmon is defined as a peak in the latter. For a small $\eta$ the two definitions agree nicely, and, in addition, $\omega_{1}$ (the real part of the complex root of $\epsilon$ ) is also very close to the zero of $\operatorname{Re} \epsilon$ (a plasmon definition found in many textbooks).

With increasing wave vector $q \neq 0$ the graphene plasmon enters the interband single-particle continuum and gets highly Landau-damped. For large $\eta$, using the appropriate definition for calculating the collective modes becomes crucial [17]. In Fig. 1, we show the real and imaginary part of the Drude loss function. While the maximum of $\operatorname{Im} \epsilon_{\mathrm{D}}^{-1}(\omega)$ is hardly effected by damping with $\eta \lesssim 1$, the zero of $\operatorname{Re} \epsilon_{\mathrm{D}}(\omega)$ is significantly lowered.

This sensitivity on $\eta$ shows the importance of using the appropriate definition for the collective

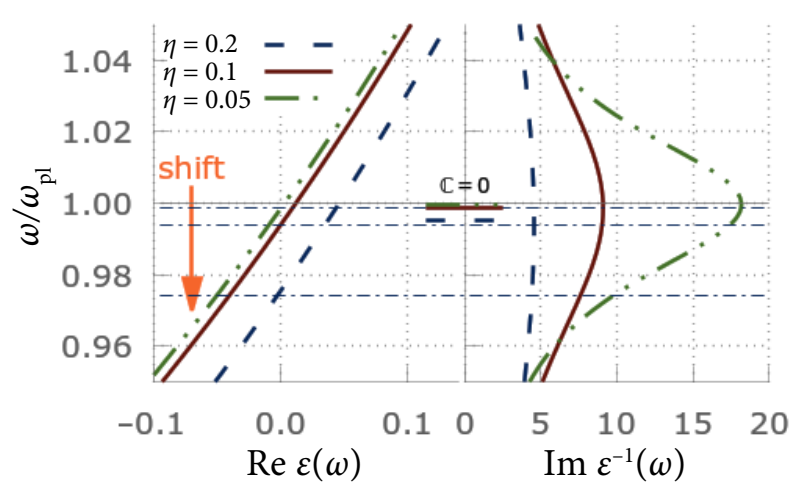

Fig. 1. The real part of the Drude dielectric function (left). The zero of $\operatorname{Re} \epsilon(\omega)$ decreases rapidly with the damping rate $\eta=\bar{\eta} \omega_{\mathrm{pl}}$. The plasmon observed in EELS is at the maximum of $\operatorname{Im} \epsilon^{-1}(\omega)$ (right). The complex root of $\epsilon(\omega)$ (a short line in the middle, marked ' $\mathbb{C}=0$ ') is affected much less. modes in spin-imbalanced graphene, where interband damping is formidable. We therefore obtain the plasmon from the maxima in the imaginary part of the response functions (condition of maximal dissipation [9]). Before presenting our results of these loss functions and that of the influence of the substrate, we briefly review the linear response theory for a homogeneous and isotropic 2DEL with linear energy dispersion, and derive the spin-resolved RPA response functions.

\section{Spin-sensitive linear response theory}

\subsection{Energy dispersion near the Dirac points}

The Pauli matrices $\underline{\underline{\sigma}}$ describe the particles' behaviour in an applied magnetic field $\mathbf{B}$, with $\underline{\underline{\sigma_{z}}}$ denoting their orientation with respect to the given direction. Similarly, two more Pauli vectors $\underline{\underline{\tau^{K}}}$ and $\underline{\underline{\tau^{\prime}}}$, termed 'pseudospin', describe the states at $K$ and $K$ '. Single-particle energies $\varepsilon_{\sigma, \tau, \mathbf{k}}^{\ell}$ are thus characterized by their band index $\ell$, spin $\sigma$ and pseudospin $\tau$, and the $2 \mathrm{D}$ wave vector $\mathbf{k}$. The latter is measured relative from $K$ and $K^{\prime}$, respectively.

A thorough first-principles study of graphene's band structure in the presence of SOC was presented in Refs. [23, 24]. Aiming at spintronics applications, a large external (or 'Rashba') SOC induced by an external electric field or magnetic adatoms is desirable. Although giving rise to band splitting near the Dirac points, this gap is rather small [23]. Therefore, using a linear energy dispersion in vicinity of $K$ and $K^{\prime}$ is a good starting point.

Denoting with $\mu$ the chemical potential and measuring all energies with respect to the Fermi energy $\mu(T=0)$ of the undoped graphene (i.e. at the meeting point of the upper and lower Dirac cone), the single-particle Hamiltonian of a charge carrier with Landé factor 2 near $K$ can then be expressed as [12]

$$
\underline{\underline{\hat{h}}}_{\sigma, K} \equiv \underline{\underline{\hat{h}}}_{K}+\underline{\underline{\sigma_{z}}} \mu_{\mathrm{B}} B_{z}-\mu,
$$

where $\mu_{\mathrm{B}}$ is Bohr's magneton and unity matrices are not spelled out explicitly. The pure graphene part, in a compact and in a matrix form, reads

$$
\underline{\underline{h}}_{K}=\hbar v_{\mathrm{F}} \hat{\mathbf{p}} \cdot \underline{\underline{\underline{\tau^{K}}}}=\hbar v_{\mathrm{F}}\left(\begin{array}{cc}
0 & \frac{1}{\mathrm{i}} \frac{\partial}{\partial x}+\frac{\partial}{\partial y} \\
\frac{1}{\mathrm{i} \partial \partial}-\frac{\partial}{\partial y} & 0
\end{array}\right),
$$


where $v_{\mathrm{F}} \approx 10^{6} \mathrm{~m} / \mathrm{s}[12]$ is the material constant and $\hat{\mathbf{p}}=\hbar \nabla / \mathrm{i}$ is the $2 \mathrm{D}$ momentum operator. A similar operator $\hat{h}_{\sigma, K^{\prime}}$ holds near $K^{\prime}$ (see Appendix A).

Both $\hat{h}_{K}^{\sigma, K}$ and $\hat{h}_{K^{\prime}}$ yield the same energy dispersion proportional $k=|\mathbf{k}|$ resulting in the eigenvalues of Eq. (1a):

$$
\begin{aligned}
\epsilon_{\sigma \tau}^{\ell}(k) & =\ell \hbar v_{\mathrm{F}} k+\sigma \mu_{\mathrm{B}} B_{z}-\mu, \\
& \equiv \ell \hbar v_{\mathrm{F}} k-\mu_{\sigma} .
\end{aligned}
$$

The only effect of the valleys being to contribute the degeneracy factor $g_{\mathrm{V}}=2$ in summations, we therefore suppress this index in the following. The dispersion (2) suggests the definition of spindependent chemical potentials $\mu_{\sigma}$ as explained in Fig. 2. These determine the maximal wave vectors $k_{\sigma}^{(\mu)} \equiv \mu_{\sigma} / \hbar v_{\mathrm{F}}$ for occupations with the spin $\sigma$ (the 'Fermi wave vectors' of each spin component). Without a magnetic field, in undoped graphene all

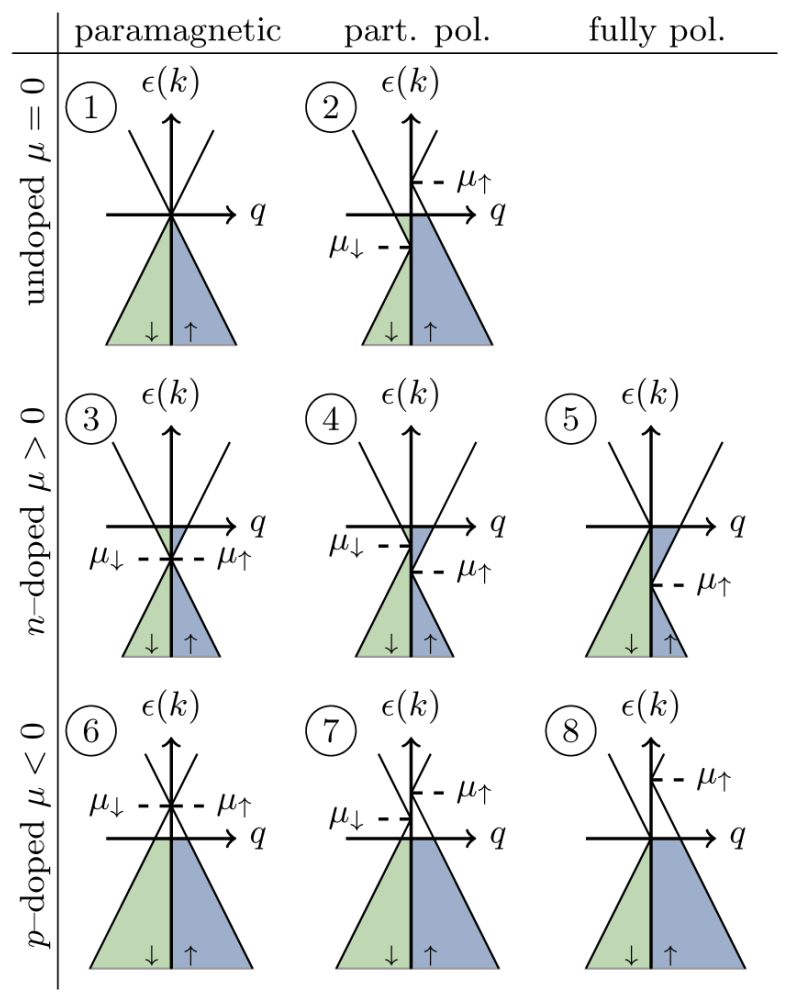

Fig. 2. Energy dispersions for all eight possible cases: undoped and doped graphene with paramagnetic, partially polarized and fully polarized settings. The left (green) cones depict the minority (or spin $\downarrow$ ) charge carriers, and the right (blue) cones show the majority (or spin $\uparrow$ ) ones. Consistent with Eq. (2), $\mu_{\sigma}(\$) 0$ shifts the crossing point to lower (higher) energies. $\mu_{\sigma}=0=\mu$, with no electrons in the conduction and no holes in the valence band. A system in $B \neq 0$ has at least one $\mu_{\sigma} \neq 0$.

The density of charge carriers with spin $\sigma$ in the conduction and valence band determines $\mu_{\sigma \tau}$ via the $T=0$ Fermi distribution function $f(\varepsilon)$ and the energies of Eq. (2):

$$
\begin{aligned}
& n_{\sigma}^{\ell}=\sum_{\tau} \int \frac{\mathrm{d}^{2} k}{(2 \pi)^{2}} f\left(\varepsilon_{\sigma \tau}^{\ell}(\mathbf{k})\right) \\
& =\frac{g_{\mathrm{V}}}{2 \pi} \int k \mathrm{~d} k \theta\left(\mu_{\sigma}-\ell \hbar v_{\mathrm{F}} k\right) .
\end{aligned}
$$

For the partial chemical potentials $\mu_{\sigma}$ there are eight different scenarios possible, corresponding to a system with the following properties:

1. undoped, paramagnetic: $\mu_{\uparrow}=0=\mu_{\downarrow}$, both spin-species have the same density $n_{\uparrow}=n_{\downarrow}$;

2. undoped, partially polarized: $\mu_{\uparrow}=-\mu_{\downarrow}, n_{\uparrow}>n_{\downarrow}$;

3. $n$-doped, paramagnetic: $\mu_{\uparrow}=\mu_{\downarrow}>0, n_{\uparrow}=n_{\downarrow}$;

4. $n$-doped, partially polarized: $\mu_{\uparrow}>\mu_{\downarrow}>0$, $n_{\uparrow}>n_{\downarrow}$;

5. $n$-doped, fully polarized: $\mu_{\uparrow}>0, \mu_{\downarrow}=0, n_{\downarrow}=0$;

6. $p$-doped, paramagnetic: $\mu_{\uparrow}=\mu_{\downarrow}<0, n_{\uparrow}=n_{\downarrow}$;

7. $p$-doped, partially polarized: $\mu_{\uparrow}<\mu_{\downarrow}<0$, $n_{\uparrow}>n_{\downarrow}$;

8. $p$-doped, fully polarized: $\mu_{\uparrow}<0, \mu_{\downarrow}=0, n_{\downarrow}=0$.

In Fig. 2 these eight cases are depicted schematically. From diagrams 5 and 8 it is seen that in these cases interband excitations are possible with zero energy.

With hindsight to spintronic applications the spin-imbalanced doped cases are of major interest. Without SOC, all the magnetic properties discussed here, in $p$ - and $n$-doped graphene behave in exactly the same manner. Without loss of generality we therefore assume zero or no doping.

The total density now equals that of the conduction band, and defines the cut-off wave vector $k_{\mu}$ as that of the paramagnetic system with this density (spin degeneracy factor $g_{\mathrm{s}}=2$ ). The maximally occupied $k_{\mu, \sigma}$ obeys analogous relations:

$$
\begin{aligned}
& n=\sum_{\sigma} n_{\sigma}^{C} \equiv \frac{g_{\mathrm{s}} g_{\mathrm{v}}}{4 \pi} k_{\mu}^{2}=\frac{g_{\mathrm{s}} g_{\mathrm{v}}}{4 \pi\left(\hbar v_{\mathrm{F}}\right)^{2}} \mu^{2}, \\
& n_{\sigma}=n_{\sigma}^{C} \equiv \frac{g_{\mathrm{v}}}{4 \pi} k_{\mu_{\sigma}}^{2}=\frac{g_{\mathrm{v}}}{4 \pi\left(\hbar v_{\mathrm{F}}\right)^{2}} \mu_{\sigma}^{2} .
\end{aligned}
$$

The polarization parameter $\zeta$ quantifies the spin imbalance in partially spin-polarized systems $(\bar{\sigma} \equiv-\sigma$ denotes the opposite spin): 


$$
\zeta_{\sigma} \equiv\left(n_{\sigma}-n_{\bar{\sigma}}\right) / n
$$

Inverting this, one readily finds $n_{\sigma}=(1+\zeta) n / 2$ and the relation of the partial Fermi wave vectors $k_{\mu, \sigma}$ and energies $\mu_{\sigma}$ with those of the paramagnetic system

$$
\begin{aligned}
& k_{\mu, \sigma}=\sqrt{1+\zeta_{\sigma}} k_{\mu}, \\
& \mu_{\sigma}=\sqrt{1+\zeta_{\sigma}} \mu .
\end{aligned}
$$

We conclude this section with noting that in order to achieve a finite density, an empirical cut-off parameter $k_{\Lambda}$ must be introduced for the valence band such that the number of states in the $\mathrm{BZ}$ is conserved [25]. In the area $A_{\mathrm{p}}=3 a^{2} / 2$ ( $a=14.2 \mathrm{~nm}$ [26]) of the primitive cell, each carbon atom contributes one p-orbital state to the valence band (as well as another one to the conduction band). This corresponds to defining $k_{\Lambda}$ from the density $n^{\mathrm{V}}=2 / A_{\mathrm{P}} \equiv \frac{g_{\mathrm{S}} g_{\mathrm{V}}}{4 \pi} k_{\Lambda}^{2}$ as a constant of the system.

\subsection{Partial Lindhard functions of graphene}

The free polarizability (or 'Lindhard function') of graphene is given by [27] (where $\mathrm{f}_{\sigma}^{\ell}(\mathbf{k}) \equiv \mathrm{f}\left(\varepsilon_{\sigma}^{\ell}(\mathbf{k})\right)$ are the Fermi functions)

$$
\begin{aligned}
& \chi_{\sigma}^{0}(q, \omega)=\frac{g_{\mathrm{V}}}{\Omega} \sum_{\ell, \ell^{\prime}, \mathbf{k}} \frac{\mathrm{f}_{\sigma}^{\ell^{\prime}}(\mathbf{q})-\mathrm{f}_{\sigma}^{\ell^{\prime}}(\mathbf{k}+\mathbf{q})}{\hbar \omega-\epsilon_{\sigma}^{\ell^{\prime}}(\mathbf{k}+\mathbf{q})+\epsilon_{\sigma}^{\ell}(\mathbf{k})+\mathrm{i} 0}+ \\
& \times\left|\left\langle\phi_{\sigma}^{\ell}(\mathbf{k}) \mid \phi_{\sigma}^{\ell^{\prime}}(\mathbf{k}+\mathbf{q})\right\rangle\right|^{2},
\end{aligned}
$$

with a small imaginary part in the denominator ensuring causality, $\Omega$ denoting the volume, and $\left|\phi_{\sigma}^{\ell}(\mathbf{k})\right\rangle$ the eigenstates of the Hamiltonian in Eq. (1a) for the band index $\ell \in\{C, V\}$. The full density-density response function is obtained by $\chi^{0}(q, \omega) \equiv \chi_{n n}^{0}(q, \omega)=\sum_{\sigma} \chi_{\sigma}^{0}(q, \omega)$.

In the following, we successively present the undoped and doped paramagnetic $\chi^{0}$ [27] and then derive the partially spin-polarized Lindhard functions. All quantities are given in reduced units, energies being measured in $\mu$ and lengths in $k_{\mu}$ of the paramagnetic 2D Dirac liquid, in particular $\bar{q}=q / k_{\mu}, \bar{\omega}=\omega \hbar / \mu$ and $\bar{\chi}=\mu \chi / n$. The alert reader may notice that these appear to diverge in the undoped system, where $\mu \rightarrow 0$. In this case, any arbitrary $\tilde{\mu}$ and $\widetilde{k}_{\mu}$ with $\tilde{\mu}=\hbar v_{\mathrm{F}} \widetilde{k}_{\mu}$ describe the same result. This reflects the fact that $\chi^{0}(q, \omega) \propto n / \mu$ which both vanish, but at a finite ratio. The chosen units allow meaningful comparisons of systems having different $\zeta$ by analogy with conventional 2DELs with parabolic dispersion.

A. Undoped system $(\mu=0)$ :

In the case of no doping, where all $\mu_{\sigma}=0$, the full free response reads [27]

$$
\begin{aligned}
& \chi_{\mu=0}^{0}(q, \omega)=\frac{g_{\mathrm{S}} g_{\mathrm{V}}}{16} \frac{q^{2}}{\hbar v_{\mathrm{F}} \sqrt{q^{2}-\left(\omega / v_{\mathrm{F}}\right)^{2}}} \\
& =\frac{g_{\mathrm{S}} g_{\mathrm{V}}}{16} \frac{k_{\mu}^{2}}{\mu} \frac{\left(q / k_{\mu}\right)^{2}}{\sqrt{\left(q / k_{\mu}\right)^{2}-(\omega / \mu)^{2}}},
\end{aligned}
$$

or, in reduced units,

$$
\bar{\chi}_{\mu=0}^{0}(\bar{q}, \bar{\omega})=\frac{1}{32 \pi} \frac{\bar{q}^{2}}{\sqrt{\bar{q}^{2}-\bar{\omega}^{2}}} \equiv \frac{n}{\mu} \chi_{\mu=0}^{0}(\bar{q}, \bar{\omega}) .
$$

The partial spin response is $\chi_{\sigma}^{0}=\frac{1}{g_{\mathrm{S}}} \chi^{0}$ due to symmetry.

B. Doped system $(\mu \neq 0)$ :

A non-vanishing chemical potential $\mu_{\sigma} \neq 0$ changes the form of the response function dramatically to

$$
\begin{aligned}
& \chi_{\mu \neq 0}^{0}(q, \omega)=\frac{g_{\mathrm{S}} g_{\mathrm{V}}}{16 \pi} \frac{k_{\mu}^{2}}{\mu}\left(\frac{\bar{q}^{2} \mathcal{F}(\bar{q}, \bar{\omega})}{\sqrt{\bar{q}^{2}-\bar{\omega}^{2}}}-8\right), \\
& \bar{\chi}_{\mu \neq 0}^{0}(\bar{q}, \bar{\omega})=\frac{1}{4 \pi^{2}}-\frac{1}{32 \pi^{2}} \frac{\bar{q}^{2} \mathcal{F}(\bar{q}, \bar{\omega})}{\sqrt{\bar{q}^{2}-\bar{\omega}^{2}}},
\end{aligned}
$$

with

$$
\mathcal{F}(\bar{q}, \bar{\omega})=\mathcal{G}^{+}\left(\frac{2+\bar{\omega}}{\bar{q}}\right)-\mathcal{G}^{-}\left(\frac{2-\bar{\omega}}{\bar{q}}\right)
$$

and $\mathcal{G}^{ \pm}(z)=z \sqrt{1-z^{2}}+\mathrm{i} \operatorname{arccosh}(z)$. The function $\mathcal{F}$ determines the structure of the response function in the $(\bar{q}, \bar{\omega})$-plane, the various arising regions characterized by $z_{ \pm} \equiv(2 \pm \bar{\omega}) / \bar{q}$ are shown in Fig. 3 .

In a spin-polarized system, the response functions of the constituents are rescaled with individual Fermi wave vectors $k_{\mu, \sigma}$. Thus we get for the partial response functions

$$
\begin{aligned}
& \chi_{\sigma}^{0}(q, \omega, \mu)=\left\{\begin{array}{l}
\frac{n}{\mu g_{\mathrm{S}}} \bar{\chi}_{\mu=0}^{0}(\bar{q}, \bar{\omega}) \text { for } \mu_{\sigma}=0 \text { (undoped) } \\
\frac{n_{\sigma}}{\mu_{\sigma}} \bar{\chi}_{\mu \neq 0}^{0}\left(\bar{q}_{\sigma}, \bar{\omega}_{\sigma}\right) \text { for } \mu_{\sigma} \neq 0 \text { (doped) }
\end{array}\right. \\
& \equiv \bar{\chi}_{\sigma}^{0}(\bar{q}, \bar{\omega}) n / \mu .
\end{aligned}
$$



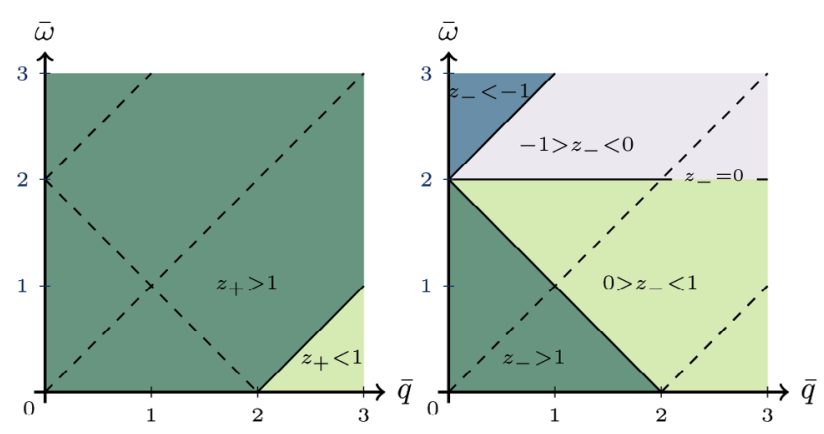

Fig. 3. Regions in the reduced $(\bar{q}, \bar{\omega})$-plane in which $z_{+}$(left) and $z_{-}$(right) are positive (green), negative (blue), and where they exceed \pm 1 .

The Fourier transform of the Coulomb interaction expressed in reduced units is $V_{\mathrm{C}}(q)=\frac{\mu}{n} V_{\mathrm{C}}(\bar{q})=2 \pi \hbar v_{\mathrm{F}} \alpha / q$ with the effective graphene coupling constant $\alpha$. For a free standing graphene sheet it is given by [27] $\alpha=\alpha_{\mathrm{gr}}=e^{2} / 4 \pi \epsilon_{0} \hbar v_{\mathrm{F}} \approx 2.2$ and by $\alpha=\alpha_{\mathrm{gr}} / \kappa_{\mathrm{s}}(\omega)$ in a surrounding with a dielectric function $\kappa_{\mathrm{s}}(\omega)$.

\subsection{Spin-sensitive Random Phase Approximation}

The observed excitations are determined by mutually dependent exchange and correlation effects. In the RPA, particles react with the free response $\chi_{\sigma}^{0}$ (ensuring the Pauli principle and thus accounting for exchange) to the effective electric and magnetic field in the probe; this mean field reflects the correlations. Generalized RPA theories use refined effective interactions $V_{\sigma \sigma^{\prime}}(q)$, various spin-dependent potentials having been introduced for the 2DEL 16 , 28-31. The generalized RPA response of multicomponent systems takes a matrix form [29]

$$
\chi=\left(1-\mathrm{V} \cdot \chi^{0}\right)^{-1} \cdot \chi^{0} .
$$

In the present case $\mathrm{V}=\left(V_{\sigma \sigma}\right)$, and $\chi^{0}=\left(\delta_{\sigma \sigma} \chi_{\sigma}^{0}\right)$. Generalizing these potentials further to dynamic ones allows one to capture double plasmon excitations [32, 33] and intrinsic damping. Except for artificial graphene [34-36], correlations here [37, 38] are well described by the 'bare' RPA, where all matrix elements are the Coulomb potential $V_{\mathrm{C}}(q)$.

We calculated the density-density, densityspin and spin-spin response functions, which can be obtained from Eq. (12):

$$
\chi_{n n}=\sum_{\sigma, \sigma^{\prime}} \chi_{\sigma, \sigma^{\prime}}=\chi_{\uparrow \uparrow}+\chi_{\downarrow \downarrow}+2 \chi_{\uparrow \downarrow},
$$

$$
\begin{aligned}
& \chi_{s s}=\sum_{\sigma, \sigma^{\prime}} \sigma \sigma^{\prime} \chi_{\sigma, \sigma^{\prime}}=\chi_{\uparrow \uparrow}+\chi_{\downarrow \downarrow}-2 \chi_{\uparrow \downarrow}, \\
& \chi_{n s}=\sum_{\sigma, \sigma^{\prime}} \sigma \chi_{\sigma, \sigma^{\prime}}=\chi_{\uparrow \uparrow}-\chi_{\downarrow \downarrow} .
\end{aligned}
$$

From the density-density response function, the dielectric function is obtained directly via

$$
\epsilon_{\mathrm{gr}}^{-1}(q, \omega)=1+V_{\mathrm{C}}(q) \chi_{n n}(q, \omega)
$$

As $\operatorname{Im} \chi_{n n}$ is proportional to the dynamic structure factor, $\operatorname{Im} \epsilon_{\mathrm{gr}}^{-1}$ gives the loss function of graphene. How the individual response functions of Eq. (14) contribute to the Raman spectrum in the 2DEL is nicely explained in [22]. Another major importance of their imaginary part is that they cause a phase delay in the response to external perturbation, thus giving rise to energy dissipation.

Our results for all three parts of graphene's loss function are shown in Fig. 4 for a doped system with $\mu=1800 \mathrm{~cm}^{-1}$ and a spin-polarization of $\zeta=0.5$ (this means that $n \approx 10^{15} \mathrm{~cm}^{-2}$ and that $75 \%$ of the spins are $\uparrow$ ). The plasmon is clearly visible in all three response functions, displaying the $\sqrt{q}$-behaviour for long wavelengths. The wave vector where it enters the interband PHB is commonly referred to as $q_{\mathrm{c}}$, beyond $q_{\mathrm{c}}$ the mode gets strongly Landau-damped [29].

We explicitly point out that both magneticfield related response functions, $\chi_{s s}$ and $\chi_{n s}$, show a distinct lack of excitations above the plasmon in the minority interband PHB (a sign change in $\chi_{n s}$ and a white region in $\chi_{s s}$ ). Due to the similarity to the 2DEL [16, 39], we call it 'magnetic antiresonance' (mAR). It can be understood as follows. An external magnetic perturbation $B^{\text {ext }}$ leads to a fluctuation in the magnetization (or spin-density $s(\mathbf{r}))$, which for $\zeta \neq 0$ due to the Coulomb coupling implies a fluctuation in the particle-density $n(\mathbf{r})$ as well: $\delta n=\chi_{n s} B^{\text {ext }}$ and $\delta s=\chi_{s s} B^{\text {ext }}$. The imaginary part of the response functions representing energy absorption, the vanishing of both, $\operatorname{Im} \chi_{s s}(q, \omega)$ and $\operatorname{Im} \chi_{n s}(q, \omega)$, prohibits magnetic dissipation at these wave vectors and frequencies (Re remains the finite). This phenomenon is similar to the well known Fano-resonance [40] and fundamental for a binary system (here, $\uparrow$ and $\downarrow$ ). 


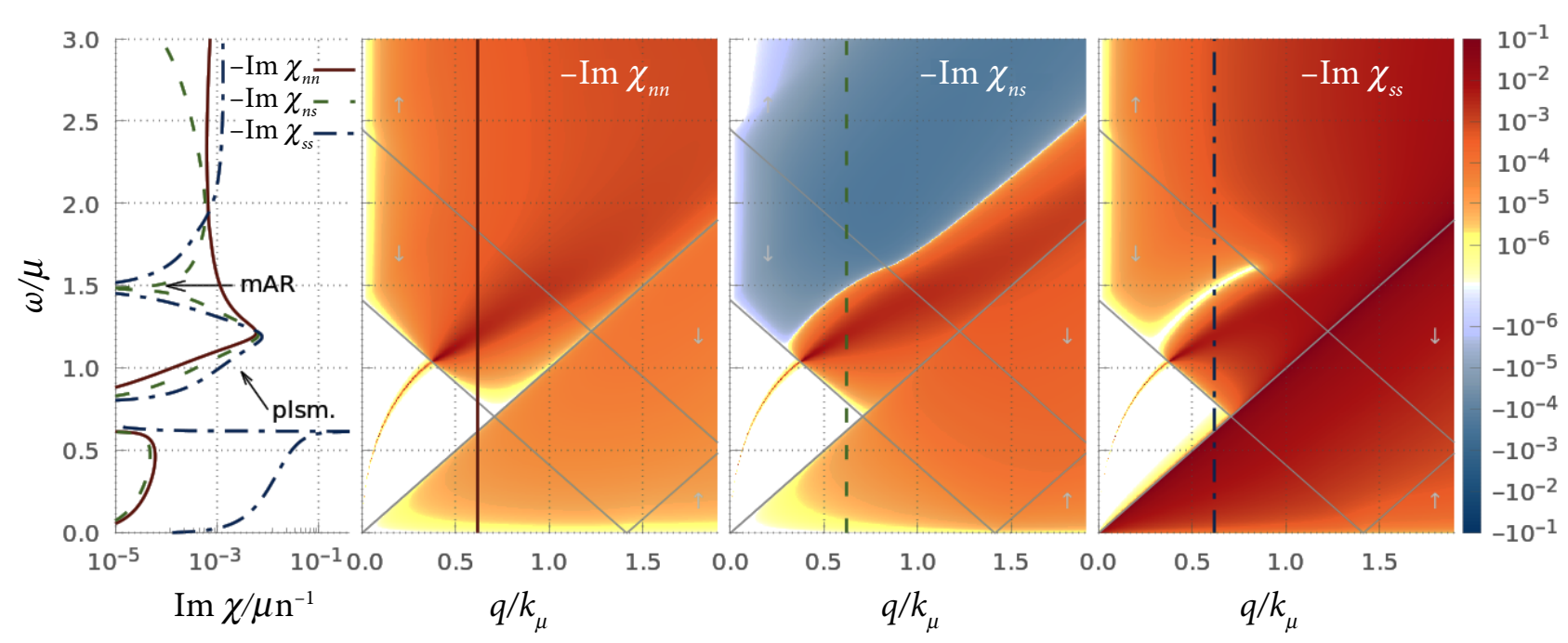

Fig. 4. The negative imaginary part of the response functions $\chi_{n n}, \chi_{n s}$ and $\chi_{s s}$ for spin polarization $\zeta=0.5$, in the $(q, \omega)$ plane (right), as well as at the experimentally accessible wave vector, $q_{0} \approx 0.62 k_{\mu}$ (left). The styles of the vertical $q_{0}$ lines match those of the same function in the left plot. The $q_{0}$ plasmon ('plsm.), having entered the interband $\mathrm{PHB}$, is strongly Landau damped. The magnetic antiresonance (mAR) [39] is the prominent curved white region in the rightmost panel; the corresponding dark-blue dash-dotted curve has a distinct gap around $\omega \approx 1.5 \mu$, and the green dashed curve becomes zero there.

\section{SNOM reflectivity}

The basic principle behind s-SNOM is to illuminate the apex of a sharp cantilever above the sample, polarizing the tip. Due to its small curvature the resulting local electric (dipole-)field is very strong. This near field then interacts with the specimen and is backscattered, sensibly changing, in turn, the amplitude and phase of the reflected light far away from the sample. Background scattering from both, the tip and the surface, is deduced by vibrating the cantilever and demodulation of the detected signal. The method provides a high spatial resolution, probing wavelengths largely independent of the illumination.

With s-SNOM, density waves can be induced and observed at much larger wave vectors compared to other optical means, $q \gg 1 / \lambda_{\text {light }}$. The accessible $q$ are in the order of the inverse of the cantilever tip radius $a$.

The s-SNOM signal strongly depends on the optical properties of the sample, with contributions from the substrate as well as from the graphene sheet. The reflectivity for the P-polarized light of the supporting material with dielectric function $\kappa_{\mathrm{S}}$ is approximated as

$$
r_{\mathrm{p}}=1-\frac{1}{\bar{\kappa}(\omega)}, \quad \bar{\kappa}=\frac{1}{2}\left(1+\kappa_{\mathrm{S}}\right) .
$$

Here, we use the results measured by Fei et al. [9], where we performed the least square fit (see Appendix B for details). Placing a graphene sheet onto this substrate changes its reflectivity to

$$
r_{\mathrm{p}}=1-\frac{1}{\bar{\kappa}(\omega)-1+\epsilon_{\mathrm{gr}}(q, \omega)}
$$

with graphene's dielectric function $\epsilon_{\mathrm{gr}}$ given in Eq. (14).

The dipole moment $p_{\mathrm{D}}$ induced in the tip is caused by the local field composed of both, the external one as well as the backscattered field of the sample (typically described by an image dipole). Denoting the polarizability as $\alpha$, this implies that $p_{\mathrm{D}}=\alpha E_{0}+G p_{\mathrm{D}}$. Here, the function $G$ for the single-dipole approximation gets relevant. For the cantilever tip at distance $d$ it reads [41, 42]

$$
\begin{aligned}
& G(\omega, d)=\int_{0}^{\infty} \widetilde{k}^{2} \exp (-2 \tilde{k} \widetilde{d}) r_{\mathrm{P}}(\tilde{k}, \omega) \mathrm{d} \tilde{k} \\
& \equiv \frac{1}{I_{\tilde{d}}} \int_{0}^{\infty} g_{\tilde{d}}(\tilde{k}) r_{\mathrm{P}}(\tilde{k}, \omega) \mathrm{d} \tilde{k},
\end{aligned}
$$

with $I_{\widetilde{d}} \equiv 1 / 4 \widetilde{d}^{3}$ and where all lengths are now conveniently measured via the tip-radius: $\widetilde{k} \equiv q a$ and $\widetilde{d} \equiv d / a$. In Eq. (17) the reflectivity $r_{\mathrm{p}}$ is basically averaged with the distribution function $g_{\tilde{d}}(\widetilde{k})=4 \widetilde{d}^{3} \widetilde{k}^{2} \exp (-2 k d)$ which has its maximum 


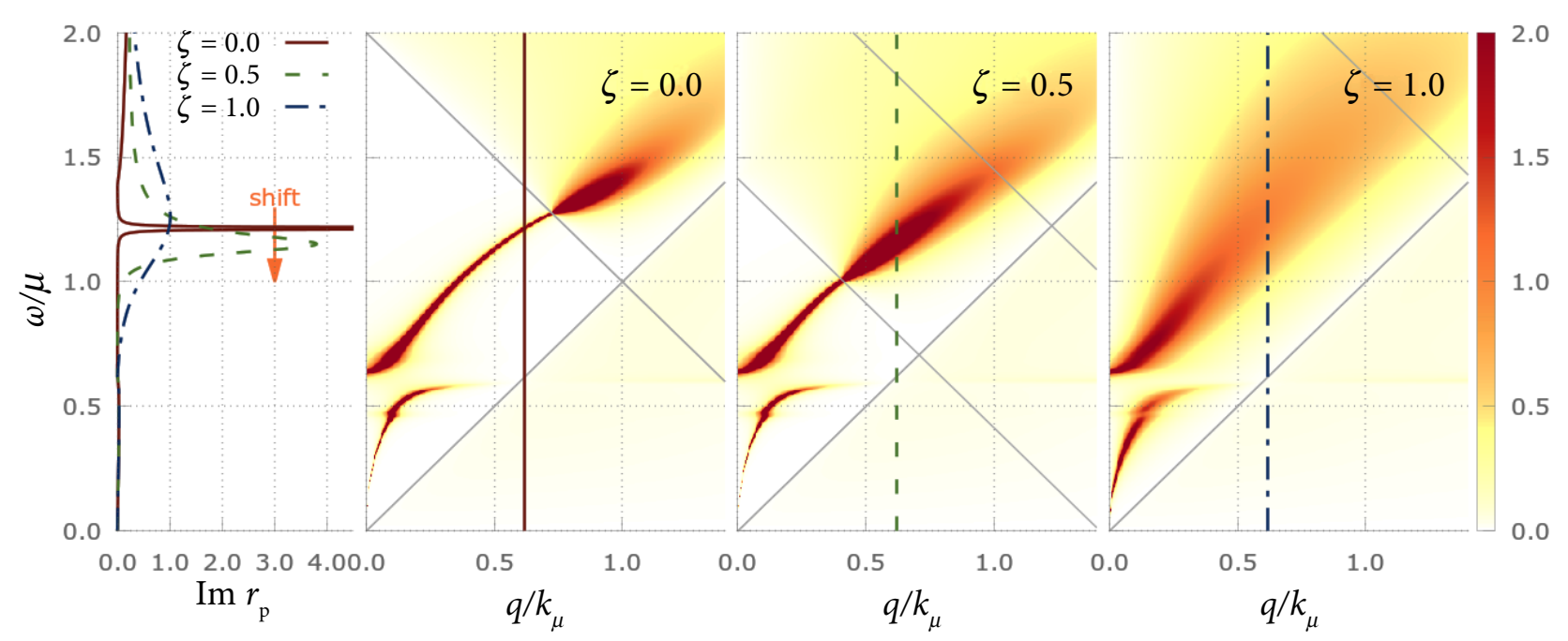

Fig. 5. The reflectivity $r_{\mathrm{p}}$ of graphene on $\mathrm{SiO}_{2}$ for zero, partial and full polarization $(\zeta=0.0,0.5$ and 1.0 , respectively). As in Fig. 4 , the left part shows cuts at $q_{0}=0.62 k_{\mu}$, a typical wave vector for s-SNOM with a tip radius of $a \approx 30 \mathrm{~nm}$. The doping level of the graphene sheet corresponds to $\mu=1800 \mathrm{~cm}^{-1}$.

at $k_{\mathrm{mx}}=1 / d$. Consequently, $G(\omega, d)$ is dominated by $r_{\mathrm{p}}(1 / d, \omega)$.

In s-SNOM, the cantilever is typically operated in the tapping mode. To improve the signal to noise ratio, the measured signal $s$ is proportional to the third order demodulation integral

$$
s_{3}(\omega) \sim \int_{0}^{2 \pi} \frac{\exp (\mathrm{i} 3 \phi)}{1-G(\omega, d(\phi))} \mathrm{d} \phi=2 \pi \mathrm{i} \sum_{j}^{\sigma} \operatorname{Res} f_{s}\left(\omega, z_{j}\right),
$$

with the time periodic distance $d(\phi)=d_{0}+d_{1} \cos (\phi)$. This turns into the sum over all residues in the unit circle of

$$
f_{s}(\omega, z) \equiv \frac{-\mathrm{i} z^{2}}{1-G(\omega, d(z))},
$$

with the analytically continued distance $d(z) \equiv d_{0}+\frac{d_{1}}{2}\left(z+z^{-1}\right)$. Therefore, the measurement is determined by the poles of $f_{s}(z)$, i.e. the zeroes of $1-G(\omega, d(z))$. Approximating the function $g_{\tilde{d}}(\widetilde{k}) \approx \delta(1 / \widetilde{d}-\widetilde{k})$ leads to a better understanding of these singularities. For the free standing graphene sheet, $\bar{\kappa}=1$, the poles are given by $\epsilon_{\mathrm{gr}}(1 / d, \omega)=1 /\left(1-a^{3} / 4 d^{3}\right)$. This shows that both, the distance and tip radius, significantly influence the measured signal $s_{3}(\omega)$, so that recovering the exact plasmon position from the measured signal is highly non-trivial. Much more promising is to evaluate $s_{3}(\omega)$ numerically from the model $r_{\mathrm{p}}(q, \omega)$ with Eqs. (16)-(18), and then compare with the measured data.
We calculated the reflectivity $r_{\mathrm{p}}$ of graphene on $\mathrm{SiO}_{2}$ for the experimentally investigated parameters [9]. The minority PHB interband edge, lower for higher spin polarization $\zeta$, causes the plasmon peak, narrow at $\zeta=0.0$, to get both broader and shifted downwards until one can no longer distinguish a well-defined collective mode. This picture also nicely demonstrates how the plasmon and the opti$\mathrm{cal} \mathrm{SiO}_{2}$ modes repel each other due to the coupling between graphene and substrate in Eq. (16).

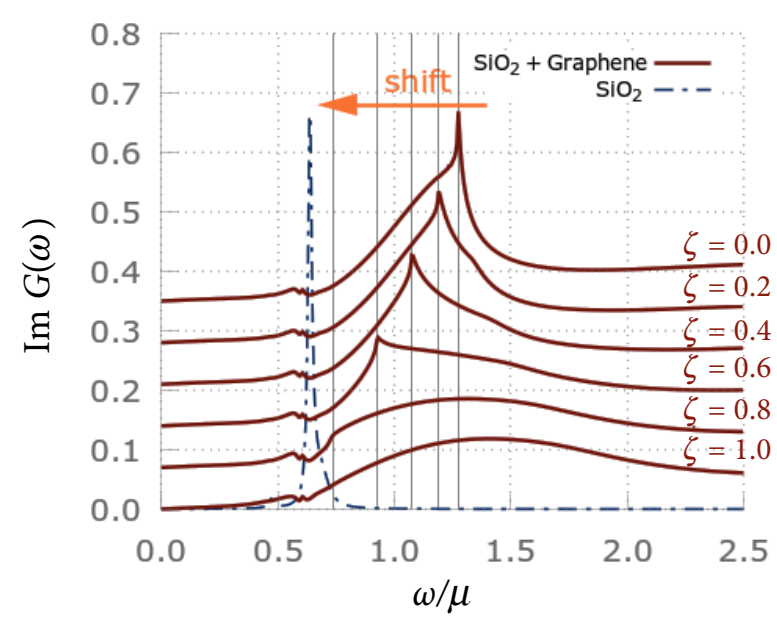

Fig. 6. The imaginary part of $G(\omega, d=0.85 a)$, Eq. (17), for different spin polarizations $\zeta$ (red solid lines) for graphene on $\mathrm{SiO}_{2}$. For low $\zeta$ the plasmon is prominent; larger $\zeta$ lead to its shift towards lower energies and rigorous damping. The narrow (blue dashed) left peak corresponds to an optical mode of the substrate without graphene. All parameters are the same as in Fig. 5 . 
For a mean doping level of $\mu=1800 \mathrm{~cm}^{-1}$, $s-S N O M$ is sensitive to wave vectors of typically $0.62 k_{\mu}$. Results for different spin polarizations $\zeta$ are shown in Fig. 6 for a tip radius of $a \approx 30 \mathrm{~nm}$.

At a polarization of $\zeta=0.6$ the plasmon mode gets strongly damped, as seen in the dipole interaction function $G(\omega, d)$ in Fig. 6. Thus, no collective behaviour can be observed anymore.

\section{Conclusions}

We have calculated the RPA linear response functions of spin-imbalanced graphene, for a freestanding sheet as well as on a silicon oxide substrate. Similar to the partially spin-polarized 2DEL, graphene also displays a magnetic anti-resonance at $\omega_{\mathrm{mAR}}(q)$. Along this characteristic line in the spectrum, an external magnetic field cannot cause excitations visible in scattering experiments: $\operatorname{Im} \chi_{\text {ss }}$ and $\operatorname{Im} \chi_{n s}$, describing spin-spin fluctuations and spin-density fluctuations, respectively, both vanish. While Im $\chi_{n s}$, containing the information on cross-correlations between charge and magnetization, just changes its sign, $\operatorname{Im} \chi_{\text {ss }}$ is essentially zero in a rather broad $\omega$ region over a wide $q$ range. Interband longitudinal spin excitations (i.e. particle-hole excitations of the minority spins with no spin-flip) are drastically suppressed and can no longer contribute to dissipation there.

Compared to the 2DEL [39], graphene's mAR shows several similarities. First, the intensity of the spin-spin and the density-spin loss function vanishes at exactly the same $(q, \omega)$ combinations, underpinning the term mAR. Second, this effect occurs in the PHB of the minority spin electrons, and, third, it starts exactly where the plasmon starts to be strongly damped: this demonstrates that the coupling between the collective mode and individual particle-hole excitations is responsible for transferring the oscillation energy from one spin species to the other. However, the mAR in graphene lies at energies above the plasmon, resulting from the fact that interband excitations are the dominating damping mechanism, in contrast to the intraband Landau damping in the 2DEL.

The conventional plasmon enters the interband PHB at the critical wave vector $q_{c}$. Increasing the spin polarization $\zeta$ lowers the minority inter- band edge and consequently also $q_{c}$, leading to an earlier Landau damping. This shifts the collective mode to lower energies and causes an intense broadening. This drastic reduction of the lifetime and mean free path of the collective mode opens the door for spin-controlled plasmon transistors. In its working point, the paramagnetic on-state has almost no mode damping, whereas in the fully polarized off-state the plasmon is thoroughly hindered.

At finite wave vectors, the dispersion can be observed with s-SNOM, in contrast to other optical setups, restricted to $q \approx 0$. In order to test our results experimentally, we therefore applied our theory to the Fresnel coefficient of P-polarized light. This reflectivity $r_{\mathrm{p}}(q, \omega)$ is a key quantity entering the dipole interaction function $G(\omega, d)$ in s-SNOM. We here predict that the plasmon peak is lowered by $\sim 30 \%$ from the paramagnetic value at $\zeta \approx 0.6$ and that it will no longer be observable for spin polarizations exceeding this value.

\section{Acknowledgements}

We thank Zhe Fei for providing us with the measured dielectric function of the substrate.

\section{Appendix A}

\section{Hamiltonian in both Dirac cones}

In the close vicinity of the $K^{\prime}$ points the pseudo-

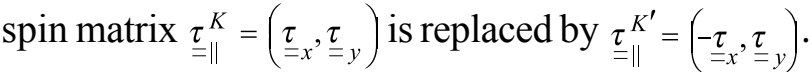
The different sign of the $x$ component can be used to define the valley quantum number $\tau= \pm 1$ and thus to write the matrix of the Hamiltonian in Eq. (1b) as

$$
\hat{h}_{=}=\hbar v_{\mathrm{F}}\left(\begin{array}{cc}
0 & \frac{1}{\frac{1}{\partial} \frac{\partial}{\partial x}+\frac{\partial}{\partial y}} \\
\tau \frac{1}{\mathrm{i}} \frac{\partial}{\partial x}-\frac{\partial}{\partial y} & 0
\end{array}\right),
$$

in order to treat the two points of the BZ simultaneously. For better clarity, in addition to their numeric values \pm 1 , we also use the following labels to distinguish the quantum numbers

$$
\begin{aligned}
& \sigma \in\{+1,-1\}=\{\uparrow, \downarrow\} \text { spin, } \\
& \tau \in\{+1,-1\}=\{+,-\} \text { valley, } \\
& \ell \in\{+1,-1\}=\{C, V\} \text { band. }
\end{aligned}
$$




\section{Appendix B}

\section{Dielectric function of silicon oxide}

Using the measured dielectric function of $\mathrm{SiO}_{2}$ obtained by Fei et al. [9, 43] we performed the least squares fit for these data to the analytic form

$$
\epsilon_{\mathrm{s}}(\omega)=\epsilon_{\infty}-g_{2}-g_{3}+\sum_{i=1}^{3} \frac{g_{i} \omega_{i}^{2}}{\omega_{i}^{2}-\omega^{2}-\mathrm{i} \omega \Gamma_{i}}
$$

with $\epsilon_{\infty}=1.85 . \epsilon_{\text {stat }}=2.27$, and $g_{1}=\epsilon_{\text {stat }}-\epsilon_{\infty}$.

Table 1. Coefficients of Eq. (B1) for the $\mathrm{SiO}_{2}$ bulk substrate (in spectroscopic units: $\mathrm{cm}^{-1}$ for $\omega_{i}$ and $\Gamma_{i}$ )

\begin{tabular}{c|ccc}
$i$ & 1 & 2 & 3 \\
\hline$g_{i}$ & & 0.029 & 0.034 \\
$\omega_{i}$ & 1072 & 845 & 1237 \\
$\Gamma_{i}$ & 29.9 & 49.8 & 147
\end{tabular}

\section{References}

[1] N.D. Mermin, Crystalline order in two dimensions, Phys. Rev. 176, 250 (1968).

[2] K.S. Novoselov, A.K. Geim, S.V. Morozov, D. Jiang, Y. Zhang, S.V. Dubonos, I.V. Grigorieva, and A.A. Firsov, Electric field effect in atomically thin carbon films, Science 306, 666-669 (2004).

[3] N. David, P. Tsvi, and W. Steven, Statistical Mechanics of Membranes and Surfaces (World Scientific, 2004).

[4] P.L. Doussal and L. Radzihovsky, Self-consistent theory of polymerized membranes, Phys. Rev. Lett. 69, 1209 (1992).

[5] S.V. Morozov, K.S. Novoselov, M.I. Katsnelson, F. Schedin, L.A. Ponomarenko, D. Jiang, and A.K. Geim, Strong suppression of weak localization in graphene, Phys. Rev. Lett. 97, 016801 (2006).

[6] A. Vakil and N. Engheta, Transformation optics using graphene, Science 332, 1291-1294 (2011).

[7] B. Yao, Y. Liu, S.-W. Huang, C. Choi, Z. Xie, J.F. Flores, Y. Wu, M. Yu, D.-L. Kwong, Y. Huang, et al., Broadband gate-tunable terahertz plasmons in graphene heterostructures, Nat. Photonics 12, 22 (2018).

[8] F. J. Garcia de Abajo, Graphene plasmonics: challenges and opportunities, ACS Photonics 1, 135152 (2014).
[9] Z. Fei, G.O. Andreev, W. Bao, L.M. Zhang, A.S. McLeod, C. Wang, M.K. Stewart, Z. Zhao, G. Dominguez, M. Thiemens, et al., Infrared nanoscopy of Dirac plasmons at the graphene- $\mathrm{SiO}_{2}$ interface, Nano Lett. 11, 4701-4705 (2011).

[10]H. Yan, T. Low, W. Zhu, Y. Wu, M. Freitag, X. Li, F. Guinea, P. Avouris, and F. Xia, Damping pathways of mid-infrared plasmons in graphene nanostructures, Nat. Photonics 7, 394 (2013).

[11]T. Low and P. Avouris, Graphene plasmonics for terahertz to mid-infrared applications, ACS Nano 8, 1086-1101 (2014).

[12]A.H. Castro Neto, F. Guinea, N.M.R. Peres, K.S. Novoselov, and A.K. Geim, The electronic properties of graphene, Rev. Mod. Phys. 81, 109 (2009).

[13]L.D. Landau, On the vibrations of the electronic plasma, Zh. Eksp. Teor. Fiz. 10, 25 (1946).

[14]S.A. Wolf, D.D. Awschalom, R.A. Buhrman, J.M. Daughton, S. von Molnar, M.L. Roukes, A.Y.Chtchelkanova, and D.M. Treger, Spintronics: a spin-based electronics vision for the future, Science 294, 1488-1495 (2001).

[15]A. Agarwal, M. Polini, G. Vignale, and M.E. Flatté, Long-lived spin plasmons in a spin-polarized two-dimensional electron gas, Phys. Rev. B 90, 155409 (2014).

[16]D. Kreil, R. Hobbiger, J.T. Drachta, and H.M. Böhm, Excitations in a spin-polarized twodimensional electron gas, Phys. Rev. B 92, 205426 (2015).

[17]R. Hobbiger, J.T. Drachta, D. Kreil, and H.M. Böhm, Phenomenological plasmon broadening and relation to the dispersion, Solid State Commun. 252, 54-58 (2017).

[18]D. Pines, Elementary Excitations in Solids (Perseus Books, Massachusetts, 1999).

[19]C. Lee, J.Y. Kim, S. Bae, K.S. Kim, B.H. Hong, and E.J. Choi, Optical response of large scale single layer graphene, Appl. Phys. Lett. 98, 071905 (2011).

[20]M. Bonitz, Quantum Kinetic Theory (Springer, 1998).

[21]C.F. Hirjibehedin, A. Pinczuk, B.S. Dennis, L.N. Pfeiffer, and K.W. West, Evidence of electron correlations in plasmon dispersions of ultralow 
density two-dimensional electron systems, Phys. Rev. B 65, 161309 (2002).

[22]F. Perez, Spin-polarized two-dimensional electron gas embedded in a semi-magnetic quantum well: Ground state, spin responses, spin excitations, and Raman spectrum, Phys. Rev. B 79, 045306 (2009).

[23]S. Abdelouahed, A. Ernst, J. Henk, I.V. Maznichenko, and I. Mertig, Spin-split electronic states in graphene: Effects due to lattice deformation, Rashba effect, and adatoms by first principles, Phys. Rev. B 82, 125424 (2010).

[24]D.V. Fedorov, M. Gradhand, S. Ostanin, I.V. Maznichenko, A. Ernst, J. Fabian, and I. Mertig, Impact of electron-impurity scattering on the spin relaxation time in graphene: A first-principles study, Phys. Rev. Lett. 110, 156602 (2013).

[25]M.W.C. Dharma-Wardana, Coulomb interactions of massless Dirac fermions in graphene; pair-distribution functions and exchange-driven spin-polarized phases, Solid State Comm. 140, 4-8 (2006).

[26]D.R. Cooper, B. D’Anjou, N. Ghattamaneni, B. Harack, M. Hilke, A. Horth, N. Majlis, M. Massicotte, L. Vandsburger, E. Whiteway, et al., Experimental review of graphene, ISRN Cond. Matt. Phys. 2012, Article ID 501686 (2012).

[27]B. Wunsch, T. Stauber, F. Sols, and F. Guine, Dynamical polarization of graphene at finite doping, New J. Phys. 8, 318 (2006).

[28]N. Iwamoto, Static local-field corrections of twodimensional electron liquids, Phys. Rev. B 43, 2174-2182 (1991).

[29]G. Giuliani and G. Vignale, Quantum Theory of the Electron Liquid (Cambridge University Press, 2005).

[30]R. Asgari, A.L. Subaşı, A.A. Sabouri-Dodaran, and B. Tanatar, Static local-field factors in a twodimensional electron liquid, Phys. Rev. B 74, 155319 (2006).

[31]J. Moreno and D.C. Marinescu, Local-field factors in a polarized two-dimensional electron gas, Phys. Rev. B 68(19), 195210 (2003).

[32]H.M. Böhm, R. Holler, E. Krotscheck, and M. Panholzer, Dynamic many-body theory:
Dynamics of strongly correlated Fermi fluids, Phys. Rev. B 82(22), 224505 (2010).

[33]M. Panholzer, M. Gatti, and L. Reining, Nonlocal and nonadiabatic effects in the charge-density response of solids: A time-dependent density-functional approach, Phys. Rev. Lett. 120(16), 166402 (2018).

[34]M. Gibertini, A. Singha, V. Pellegrini, M. Polini, G. Vignale, A. Pinczuk, L.N. Pfeiffer, and K.W. West, Engineering artificial graphene in a two-dimensional electron gas, Phys. Rev. B 79, 241406 (2009).

[35]M. Polini, F. Guinea, M. Lewenstein, H.C. Manoharan, and V. Pellegrini, Artificial honeycomb lattices for electrons, atoms and photons, Nat. Nanotechnol. 8, 625 (2013).

[36]S. Wang, D. Scarabelli, L. Du, Y.Y. Kuznetsova, L.N. Pfeiffer, K.W. West, G.C. Gardner, M.J. Manfra, V. Pellegrini, S.J. Wind, et al., Observation of Dirac bands in artificial graphene in small-period nanopatterned GaAs quantum wells, Nat. Nanotechnol. 13, 29 (2018).

[37]Note 1 . The parameter $r_{\mathrm{s}}$, termed $\alpha$ in graphene, has the constant value of approx. 2.2.

[38]Y. Liu, R.F. Willis, K.V. Emtsev, and T. Seyller, Plasmon dispersion and damping in electrically isolated two-dimensional charge sheets, Phys. Rev. B 78, 201403 (2008).

[39]D. Kreil, C. Staudinger, K. Astleithner, and H.M. Böhm, Resonant and anti-resonant modes of the dilute, spin-inbalanced, twodimensional electron liquid including correlations, Contrib. Plasm. Phys. 58, 179-188 (2018).

[40]U. Fano, Sullo spettro di assorbimento dei gas nobili presso il limite dello spettro d'arco, Nuovo Cimento 12, 154-161 (1935).

[41]F.J. Garcia de Abajo, Colloquium: Light scattering by particle and hole arrays, Rev. Mod. Phys. 79, 1267 (2007).

[42]J. Aizpurua, T. Taubner, F.J. García de Abajo, M. Brehm, and R. Hillenbrand, Substrateenhanced infrared near-field spectroscopy, Opt. Expr. 16, 1529-1545 (2008).

[43]Z. Fei (private communication). 
POLIARIZUOTO GRAFENO ANT PADĖKLO KOLEKTYVINĖS MODOS POSLINKIS PAGAL SUKINIUI JAUTRAUS ATSAKO TEORIJĄ

D. Kreil, M. Haslhofer, H.M. Böhm

Johaneso Keplerio universiteto Teorinès fizikos institutas, Lincas, Austrija 